\title{
SOME REMARKS ON THE STATICS AND DYNAMICS OF MAGNETIC FIELD STRUCTURE DEVELOPMENT IN ACTIVE REGIONS
}

\author{
V. BUMBA and J. SUDA \\ Astronomical Institute of the Czechoslovak Academy of Sciences, Ondřejov, Czechoslovakia
}

\begin{abstract}
Some comments are given concerning the fine structures in the umbra and penumbra of sunspots and their changes on the basis of high resolution photographs.
\end{abstract}

\section{Introduction}

During recent studies of the large-scale distribution of solar magnetic fields the question of the mode of magnetic field motion in the solar photosphere arose for us once again (the problem: when is it the real transport of lines of force and when the intensification of pre-existing fields). The same investigation brought us some experience about how important is the manner of illustration of the features studied. For example, sometimes it is enough to separate in synoptic charts of magnetic fields both polarities to see just different regularities.

The investigation of magnetic field motions can be made on the most concentrated fields in sunspots. To see better the details we can use good photographs of sunspots in integrated light with a resolution better than $1^{\prime \prime}$ giving us a picture of magnetic field distribution with the aid of darker features on the positives of the photosphere, as is now generally accepted. This is the way we are accustomed to study the question. But looking at the negatives of sunspot pictures we may see many more details usually lost on positives between bright photospheric and penumbral formations. And so we would like to add a few remarks to the problem of the statics and dynamics of magnetic fields in sunspots on the basis of photographic material taken during the last year (1969) with our Clark refractor $(20.5 \mathrm{~cm}$ in diameter) in a relatively narrow spectral region of about $100 \AA$ around $\lambda 5890 \AA$ with an exposure of the order of $0.001 \mathrm{~s}$., assuming that the bright features on our negatives coincide to the smallest details with the fine-structure of the magnetic field distribution in sunspots. Because of the lack of time we shall concentrate our preliminary results on one sunspot group only: it is the large group in the southern hemisphere of the Sun, first seen on July 1 and last seen on July 13, 1969 (C.M.P. July 7).

\section{Magnetic Field Fine Structure}

A view of our sunspot negative at once shows the most interesting behavior of the magnetic field : never can we see a concentration of magnetic field in the form of a dark (on negative bright) dot. Always it has the form (with the exception of nuclei of very large and dark spots) of intensified intergranular space with greater concentration in 
the crossings of this space. This 'lacy' structure with bright granular-like grains (bright umbral dots) inside the open spaces of this delicate network is striking, and certainly it is one of the main characteristics of magnetic field finest-scale distribution. The width of individual fibrils of the network is often only a few hundred kilometers; they are just observable with our instrument (Figures 1, 2, 3, 4, 5). It is a situation we can probably not simulate in the laboratory, where one usually has the plasma held inside a magnetic vessel. Here the situation seems to be reversed; the lines of the magnetic field are pushed out from the photospheric plasma forming the 'lacy' structure, sweeping through the individual channels of the network out from the main nucleus.

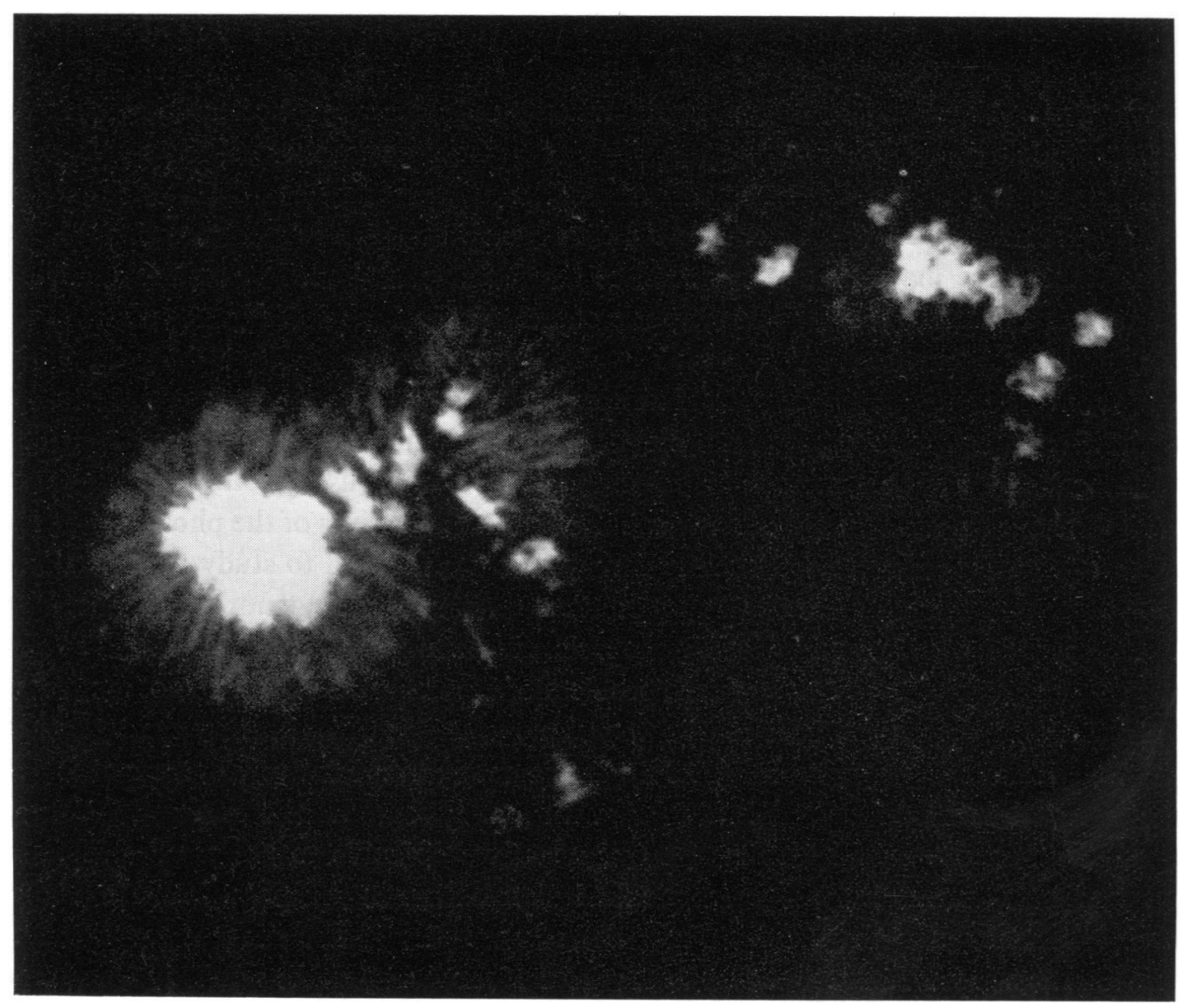

Fig. 1. Negative of the sunspot group investigated during the PFP; July 2, $\left.1969^{\left(7^{\mathrm{h}}\right.} 17^{\mathrm{m}} \mathrm{UT}\right)$.

Often the smaller spot nuclei, not very dark, consist of such 'lacy' structures or have at least in a part of their peripheries such structure. Individual fibrils of the structure continue in dark fibrils (on negatives bright) of penumbra and go on to individual fibrils of intergranular space in the photosphere surrounding the spot (Bumba, 1965), (Figures 1, 2, 3, 4, 5). On negatives the magnetic bright fibrils of the penumbra always 


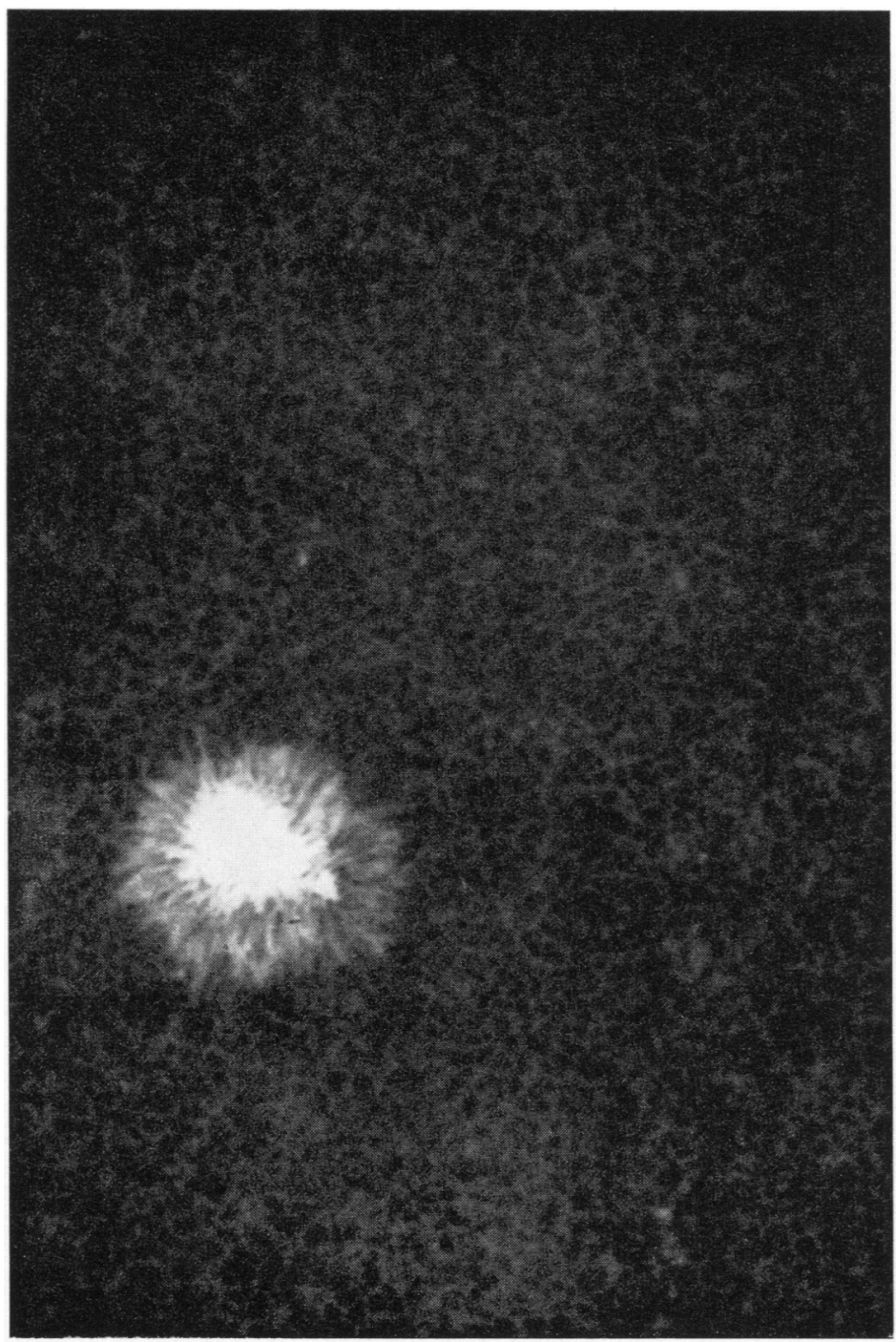

Fig. 2. Negative of a diminishing spot; July $3,1969\left(5^{\mathrm{h}} 48^{\mathrm{m}}\right.$ UT).

seem to be predominantly pressing between themselves small elongated dark areas (on positives bright) which seem to be the remains of photospheric granules.

\section{Motions}

On the spot shown we can demonstrate some rules of the development we may find on the majority of large complex spots. The spot came from the invisible disk of the Sun in the form of a large drop. During its course on the visible disk it developed two 




Fig. 3. Negative of the large complex spot investigated in the research note; July $\left.3,1969^{(\mathrm{h}} 42^{\mathrm{m}} \mathrm{UT}\right)$. 


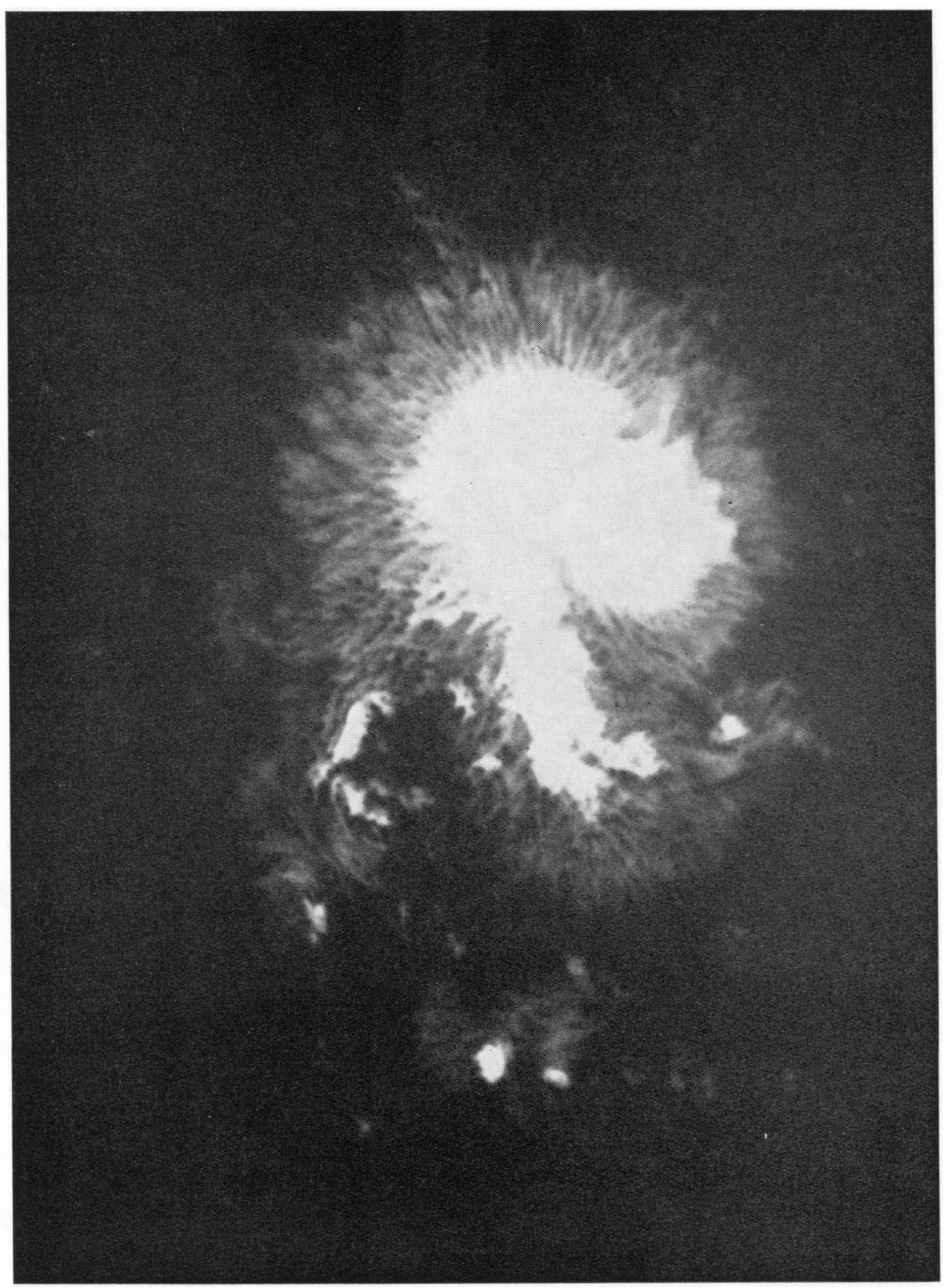

Fig. 4. The same spot on July $5,1969\left(8^{\mathrm{h}} 44^{\mathrm{m}} \mathrm{UT}\right)$. 


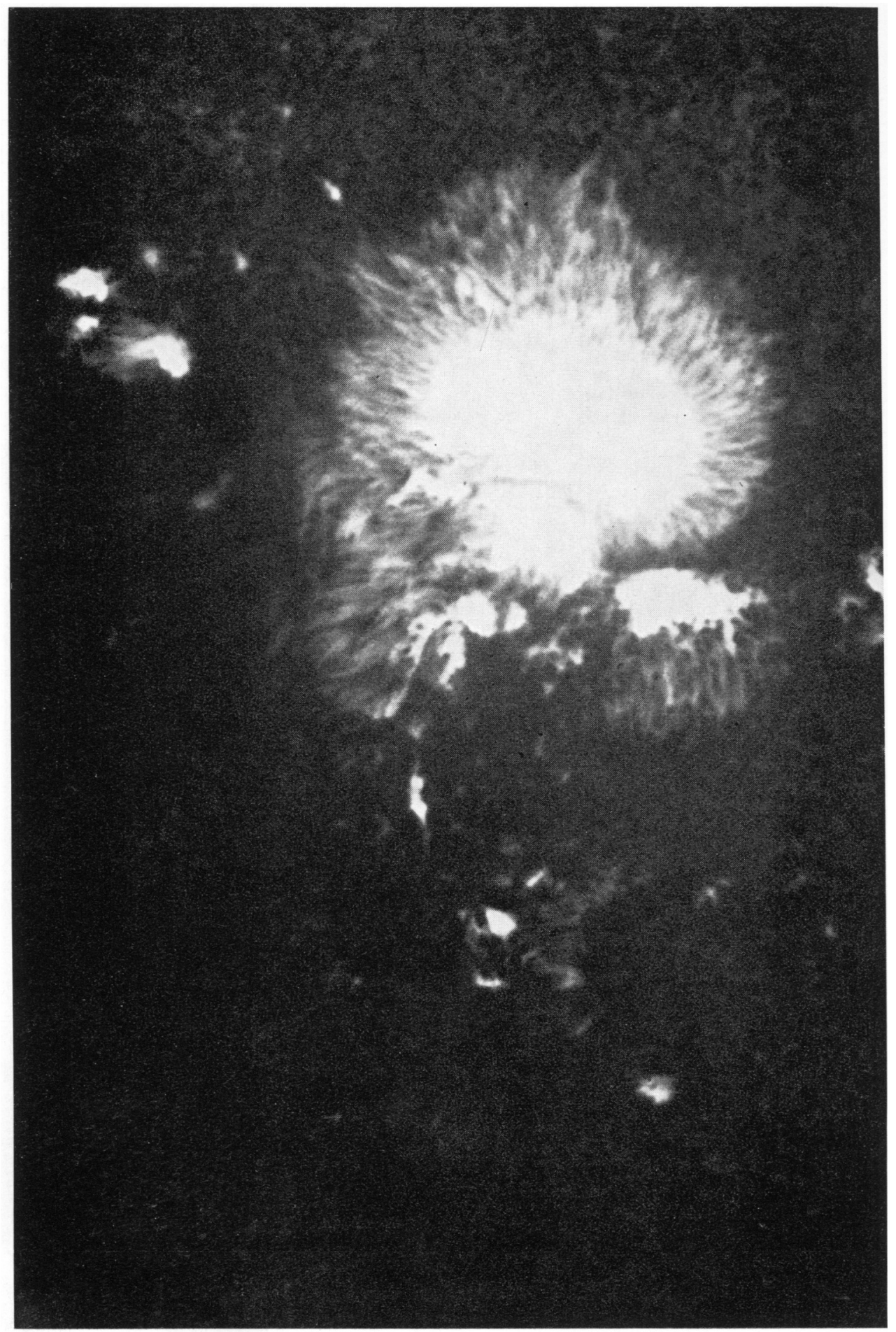

Fig. 5. Same spot on July $7,1969^{\left(8^{\mathrm{h}} 07^{\mathrm{m}}\right.}$ UT). 
THE STATICS AND DYNAMICS OF MAGNETIC FIELD STRUCTURE DEVELOPMENT
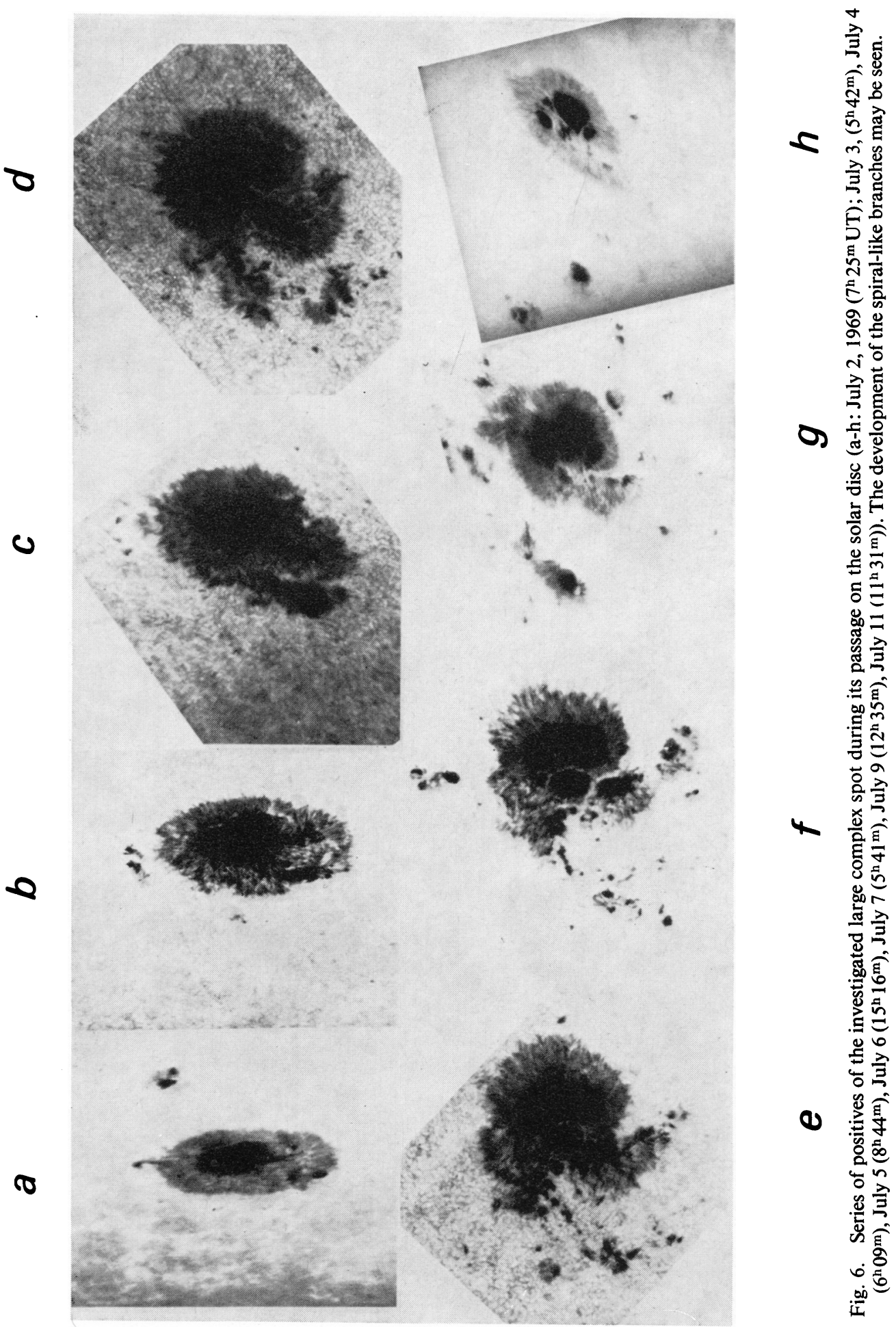

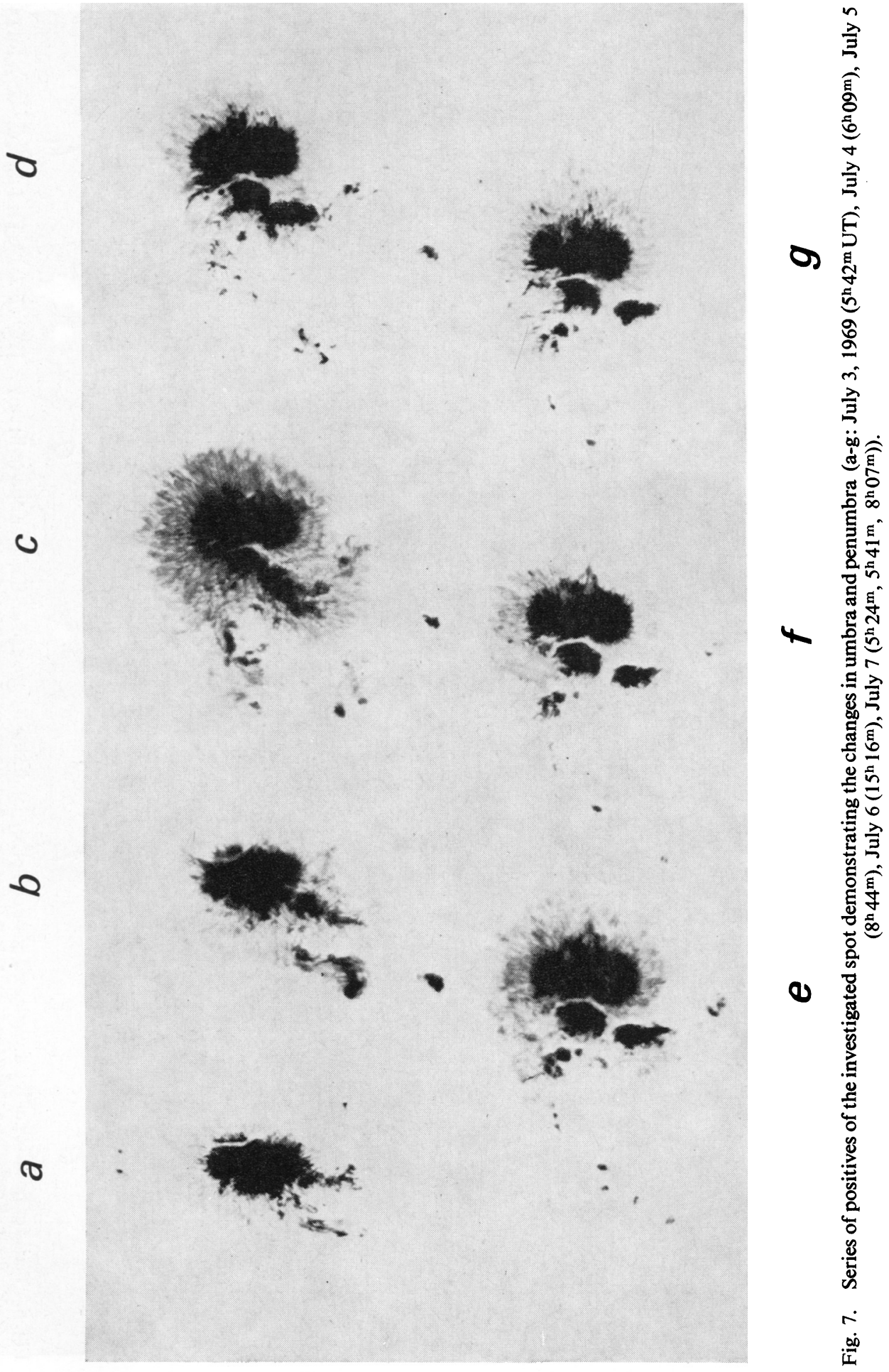

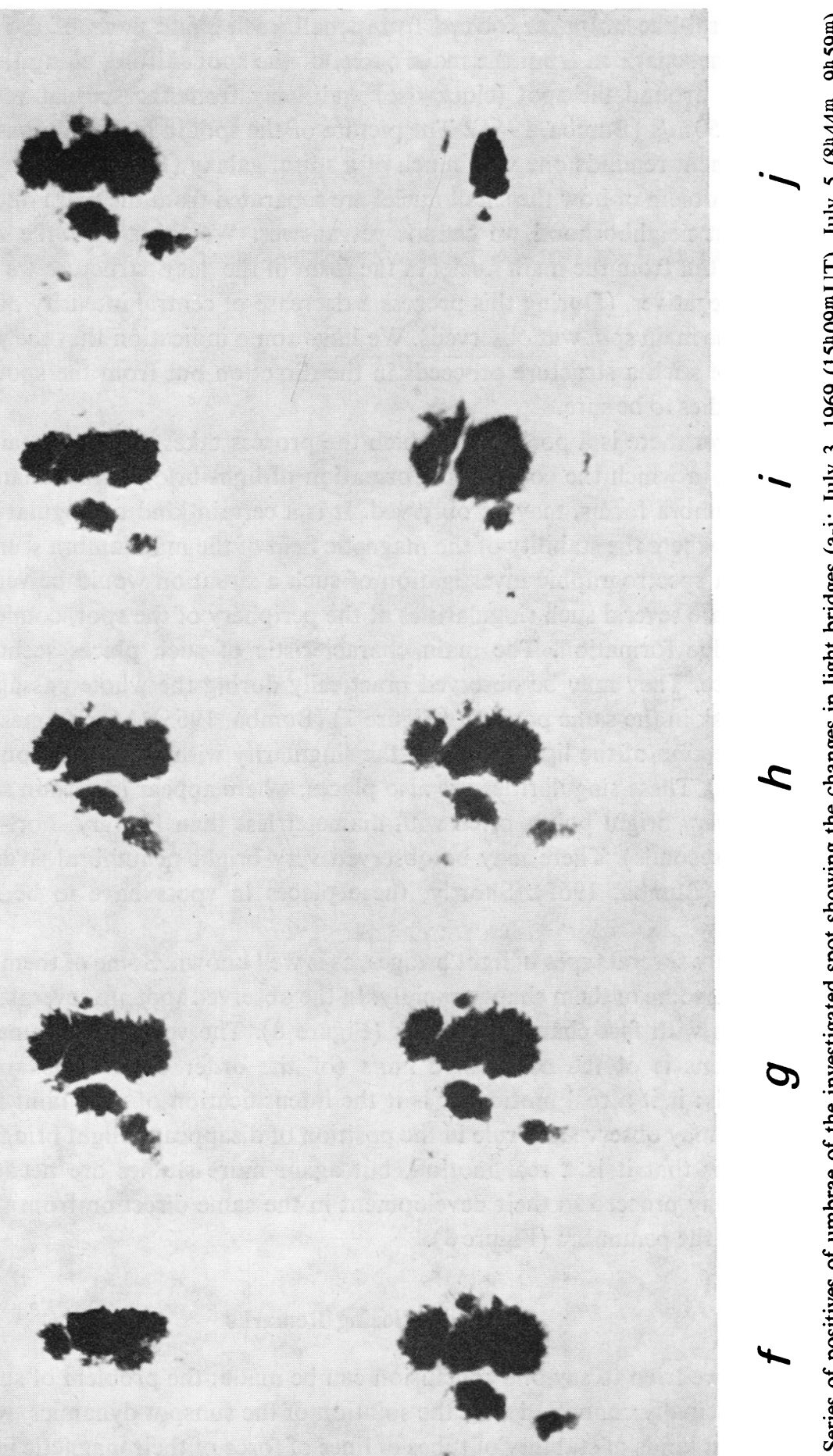

点

бิ

芒

。ํํำ



- $\stackrel{2}{\frac{2}{3}} \frac{2}{3}$

葛参

षิ

品

음

$u$

2

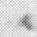

劳

क्ष

- 胥 $\frac{\Xi^{\circ}}{7}$

焉

o)

绐

ถี

है

ธัँ

क

ㅈ

总

可

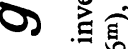

톤

5

हैํㅕㄹ

4 
main spiral-like branches formed from small nuclei, and parts of the 'rudimentary' penumbra separated from the main nuclei of the spot shifting along these spiral-like branches around the spot (clockwise) and away from the spot at velocities of the order of $50 \mathrm{~m} / \mathrm{s}$ (Bumba, 1964). The picture of the spot in maximal phase of branches development reminds one very much of a spiral galaxy (Figure 6).

The problem of how the small nuclei are separated from the main one or formed in its closest neighborhood we cannot yet answer. We think that the magnetic field squeezes out from the main nuclei in the form of the 'lacy' structure we demonstrated on our negatives. (During this process a decrease of central intensity of the magnetic field in the main spot was observed). We have some indication that the process of formation of such a structure proceeds in the direction out from the spot but we need more studies to be sure.

However there is a position in which the process takes place: the tail of the drop, the point in which the continuous formation of light bridges, fast changes in umbra and penumbra forms, may be observed. It is a certain kind of singularity in the spot, the place where the stability of the magnetic field of the main umbra seems to be diminished. A spectrographic investigation of such a situation would be very useful.

There are several such singularities at the periphery of the spot, connected with the light bridge formation. The main characteristic of such places seems to be their persistence. They may be observed practically during the whole passage of the spot on the disk in the same position (Figure 7) (Bumba, 1965). Many times one observes the connection of the light bridge in the singularity with formations outside the spot (Figure 6). These singularities are also places, where appear in certain stage of development very bright points often with diameter less than $1^{\prime \prime}$, very short-living (of the order of seconds). There may be observed very bright penumbral structures lasting for days (Bumba, 1965). Shortly, these places in spots have to be studied more carefully.

There are several types of light bridges, as is well known. Some of them are relatively persistent, some of them change rapidly. In the observed spot are several singularities the places with fast changing bridges (Figure 8). The velocity of some light bridge progressions is of the order of $1 \mathrm{~km} / \mathrm{s}$ (of the order of Evershed motions). The problem is: is it a real motion or is it the intensification of very faint bright grains, which we may observe as a rule in the position of disappeared light bridges? There are indications that it is a real motion, but again more studies are needed. The light bridges may proceed in their development in the same direction from the penumbra as toward the penumbra (Figure 8).

\section{Closing Remarks}

From all we tried to say one conclusion can be made: the problem of sunspots seems to be principally connected with the solution of the sunspot dynamics, with the study of different kinds of stability of tubes of lines of force of their magnetic fields and with the investigation of plasma-field relations in the fine-scale distribution. The greatest 
difficulty of such research is connected with the smaller resolution of practically all direct magnetic methods inside the sunspots in comparison with short-exposure photographs of sunspots used as an indirect method.

\section{References}

Bumba, V.: 1964, Prace Wroclawskiego tow. naukowego, Seria B, No. 112, p.31.

Bumba, V.: 1965, in R. Lüst (ed.), 'Stellar and Solar Magnetic Fields', IAU Symp. 22, 192. 